\title{
Integer ambiguity solution based on artificial swarm algorithm
}

\author{
Shugang Liu ${ }^{1, a^{*}, \text { Yajing Zhang }}{ }^{2, b}$ \\ ${ }^{1,2}$ Department of Computer Science,North China Electric Power University,Baoding, Hebei \\ Province, China \\ a Isg69@qq.com, b18331126515@163.com
}

Keywords: integer ambiguity LAMBDA artificial fish algorithm

Abstract. One of the keys to precise position of using double difference carrier phase measure is to resolve integer ambiguity. Least-square ambiguity decorrelation adjustment(LAMBDA) is relatively fast and precise in integer ambiguity resolution of double difference carrier phase measurement. The LAMBDA consist of two aspects: decorrelation and search. In this paper, the artificial fish swarm algorithm(AF), a global optimization algorithm, will be used for search to obtain effective integer ambiguity. Simulation data are processed by the proposed algorithm using MATLAB and the results show that the integer ambiguity resolution method based on AFSA has high search efficiency and strong robustness.

\section{Introduction}

LAMDBA introduced by P.J.G. Teunissen(see[1]) has been used widely to integer ambiguity resolution. In general, this algorithm is performed in two steps. First float solution and the corresponding variance-covariance matrix, which is computed by means of the least-squared estimation, are decorrelated by means of Z-transformation. Then the integer ambiguity can be resolved by search over an ellipsoidal region. In the process of search all the integer estimate candidates must be tested. Therefore, when the dimension of ambiguity is large, the searching space will be expanded, and increase the searching time will be increased.

In the field of artificial, global optimization algorithms such as genetic algorithm(GA) [2], simulated annealing algorithm[3], ant colony algorithm[4], artificial fish swarm algorithm(AFSA) [5] could solve the problem above better because of their parallel search. And GA is applied to search minimization of ambiguity(see [6,7]). In the current work, AFSA is introduced into integer ambiguity search by us for the first time. Referring to the integer features of ambiguity, this paper improve the artificial fish swarm algorithm by integer the status of artificial fish.

\section{Integer ambiguity estimate model}

Acrossing to the double difference carrier phase measure, the linearized observation model can be written as:

$$
V=A a+B b+\varepsilon
$$

Where $V$ is the vector of double difference carrier phase observation, $a$ is the vector of unknown integer ambiguity, $b$ is the unknown baseline vector, $A$ and $B$ are both design matrices, $\varepsilon$ is the error vector. The unknown parameters $a$ and $b$ can be computed based on (1). Least-squares estimate is used to solve the problem. The least-squares criterion is represented as:

$$
\min \|V-A a-B b\|_{Q_{V}}^{2}
$$

Where $\|\cdot\|_{Q v}^{2}=(\bullet)^{*} Q_{v}^{-1}(\bullet), Q_{v}$ denotes the variance- covariance matrix of observation. And the float solution $\hat{a}$ and $\hat{b}$, together with their variance-covariance matrices $Q_{\hat{a}}$ and $Q_{\hat{b}}$ can be obtained by means of the weighted least squares estimate. Then the integer least-squares ambiguity estimation is reduced to solve the minimization problem 


$$
J(a)=\min \|\hat{a}-a\|_{Q_{\hat{a}}}^{2}
$$

\section{Artificial fish swarm algorithm and its application to the integer ambiguity resolution}

In a water area, fishes tend to swarming to the place where foods are most abundant. And inspired by behaviors of fish swarm, AFSA was proposed to solve the optimization problem. The update strategies is obtained through the following three behaviors: preying, swarming and following.

Before applying the idea of AFSA to integer ambiguity solution, some definitions should be introduced as follows: the state of an artificial fish can be written as, $X=\left(x_{1}, x_{2}, x_{3}, \ldots, x_{n}\right)$, where $x_{i}(i=1, \ldots, n)$ is the control variable, and $\mathrm{n}$ is the dimension of ambiguity; The food concentration in current state is express as $Y=f\left(X_{i}\right)$, where $Y=f\left(X_{i}\right)$ is the objective function of optimization problem; The perception range of an artificial fish is denoted by Visual; Step represents the maximum step size a make in one movement; The distance between $i$ th and $j$ th fish is defined as $d_{i j}=\| X_{i}$ - $X_{j} \| ; \delta$ represents Congestion degree factor; The maximum try times of the artificial fish preying is described as try_number; In order to record the best state of the artificial fish swarm, call-board is used.

Taking into account the optimization efficiency, the objective function in this paper is written as :

$$
f(a)=b-\log (J(a))
$$

Where $b$ is a large positive number so that $f(a)$ is non-negative. So minimization problem of (3) is converted for the maximization problem of (4).

Initialization. Normally, the fixed solution of integer ambiguity is very closed to the float solution of it. And the success ratio to the resolve the integer ambiguity is subject to normal distribution. In this paper, in order to reduced the baseline constraint, we use a set of random numbers obeying normal distribution $\mathrm{X} \sim \mathrm{N}\left(\hat{a}, Q_{\hat{a}}\right)$ to be as initial value.

Random behavior. An artificial fish will move a step randomly in its visual rang when the searching result is not well. The next states of artificial fish is expressed as:

$$
X_{j}=X_{i}+[\operatorname{Rand}(0,1) \times \text { Step }]
$$

Preying behavior(AF-prey). Artificial fish can swim to the place where food is enough in the field of perception range. Inspired by this, preying behavior was proposed to find the optimal solution of the individual. Suppose that the current states of an artificial fish is denoted by $X_{i}$ and the next state it will move to is represented by $X_{j}$, together with corresponding food concentration $Y_{i}$ and $Y_{j}$ respectively. If $Y_{i}>Y_{j}$, the artificial fish make a integer movement towards $X_{j}$ to get a better position. Otherwise, a new state $X_{j}$ should be selected again and judge whether the condition is satisfied to make a movement. If the try times reaches maximum number try_number, random behavior will be executed.

$$
X_{j}=X_{i}+\left[\frac{\operatorname{Rand}(0,1) \times \text { Step }}{\left\|X_{j}-X_{i}\right\|}\right]\left(X_{j}-X_{i}\right)
$$

Swarm behavior(AF-swarm). An artificial fish moves to the center of the other artificial fishes in the range of sensing, and avoids overcrowding. Suppose that $X_{i}$ is the ith artificial fish. The central position is expressed as $X_{c}$. $n f$ is the number of the partner. If $Y_{j} / n f>\delta Y_{i}$, the artificial fish make a integer movement towards $X_{c}$ to get a better position. Otherwise, preying behavior will be executed. The mathematical model is:

$$
X_{j}=X_{i}+\left[\frac{\operatorname{Rand}(0,1) \times \text { Step }}{\left\|X_{c}-X_{i}\right\|}\right]\left(X_{c}-X_{i}\right)
$$


Following behavior(AF-following). The process of following behavior is to find the optimal direction of the adjacent partners in visual range. Suppose that $X_{i}$ is the ith artificial fish. $X_{j}$ is the state of the greatest partner in sensing range. If $Y_{j} / n f>\delta Y_{i}$, the artificial fish moves a integer step towards the direction of $X_{j}$. Otherwise, the artificial fish performs preying behavior. The mathematic model is:

$$
X_{j}=X_{i}+\left[\frac{\operatorname{Rand}(0,1) \times \text { Step }}{\left\|X_{j}-X_{i}\right\|}\right]\left(X_{j}-X_{i}\right)
$$

Behavior selection. According to the maximization problem of (8), each fish simulates following behavior and swarm behavior respectively. And calculate the food concentration after movement $Y_{\text {swarm }}$ and $Y_{\text {following. If }} Y_{\text {swarm }}>Y_{\text {following }}$, swarm behavior will be performed. Otherwise, following behavior will be performed.

Based on AFSA, the steps to solve integer ambiguity are set up as follows:

Step1 Based on the measurement of carrier phase, the differential equation is established. Then, the float solution of ambiguity and the relevant variance-covariance matrix are calculated by least-square method.

Step2 The process of inverse integer Cholesky decorrelation is performed. And the search space is determined with baseline length.

Step3 Initialize the states of fishes and other relevant paramenters. The best state among the fish swarm is recorded.

Step4 Perform the artificial fish swarm algorithm. And the optimal value is obtained.

Step5 Finally, according to the following equations, the integer ambiguity is obtained.

\section{Numerical results and discussions}

The parameters of the AFSA is set as follows. The number of artificial fishes is set to 50. The iteration number is 200 . Considering the integer feature of ambiguity, the step length is set to 1 . The perception range is 10 . The maximum try number of the artificial fish during preying process is set to 100. The congestion degree factor $\delta$ is 0.168 . The data instance of ambiguity float solution and the corresponding variance-covariance matrix are as follows:

$$
\hat{z}=\left[\begin{array}{c}
-4.57 \\
10.02 \\
2.35
\end{array}\right] \quad Q_{\hat{z}}=\left[\begin{array}{ccc}
4.476 & 0.334 & 0.230 \\
0.334 & 1.146 & 0.082 \\
0.230 & 0.082 & 0.626
\end{array}\right]
$$

In reference [7], a simple genetic algorithm is used to solve integer ambiguity. And compared with LAMBDA, simple genetic algorithm has higher searching efficiency. Adaptive genetic algorithm(AGA) is used in reference [6] to solve integer ambiguity. Results show that to get the same calculating precision, adaptive genetic algorithm is much faster than LAMBDA and simple genetic algorithm. In order to verify the performance of the artificial fish algorithm, the artificial fish swarm algorithm and adaptive genetic algorithm are simulated, and the results are compared. 


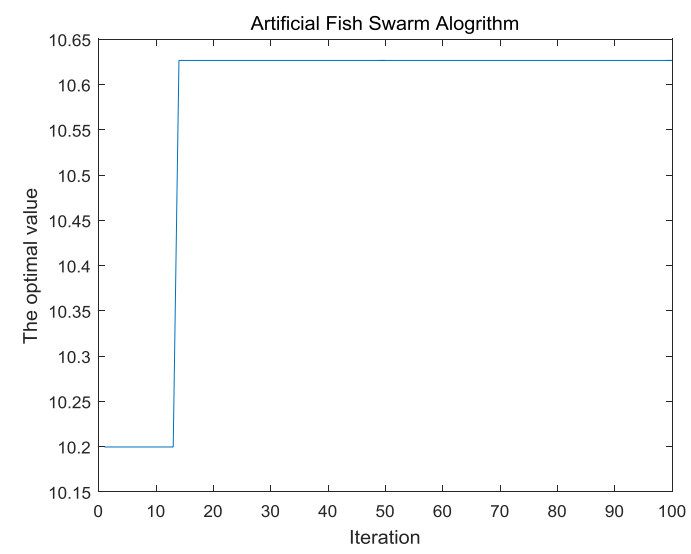

Fig.1 evolution curve of the optimal value using artificial fish swarm algorithm

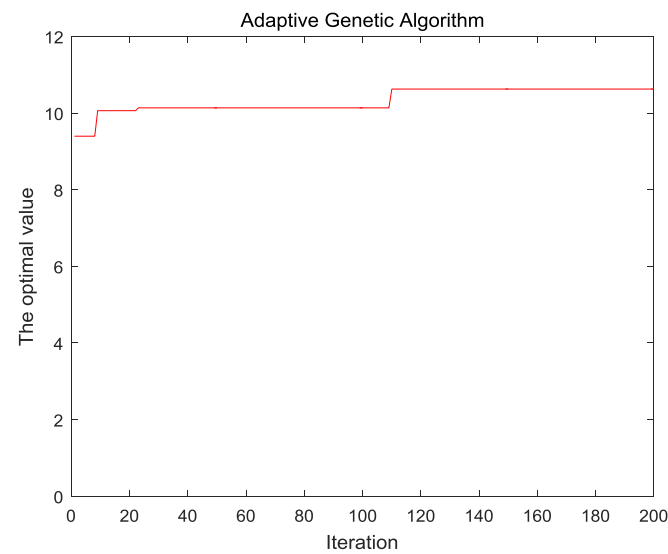

Fig. 2 evolution curve of the optimal value using adaptive genetic algorithm

In order to facilitate the comparison, we set the population quantity of genetic algorithm to be 50, the maximum iteration time to be 200. Figure 1 to figure 2 are the evolution curve of the optimal value by solving integer ambiguity using AGA and AFSA. As we can see, compared with AGA, the convergence speed of AFSA is obviously much faster. This is due to the reason that the random behavior effectively prevent local optimization. And other behaviors can accelerates the convergence speed.

\section{TABLE I}

THE OPTIMIZATION RESULT OF INTEGER AMBIGUITY AND THE SETTLEMENT TIME

\begin{tabular}{ccc}
\hline True Value & AFSA & AGA \\
\hline 5 & 5 & 5 \\
3 & 3 & 3 \\
4 & 4 & 4
\end{tabular}

Time(s) $\quad 6.374952 \quad 12.920628$

Table 1 is the optimization result of integer ambiguity and the settlement time using AFSA and AGA. As we can see that the optimal value can be obtained by using the two algorithm. But the settlement time using AFSA is less than that using AGA. In this comparative study, AFSA to solve integer ambiguity has better reliability, robustness and good value.

\section{Acknowledgment}

A novel design method for solving integer ambiguity is proposed and demonstrated. Artificial fish swarm algorithm is used in this method. In this algorithm integer ambiguity vector is mapped to the state vector of an artificial fish. Therefore, the best state of the artificial fish swarm is what we want. The result proves that this method has higher reliability and robustness. 


\section{References}

[1] P.J.G. Teunissen. The least-square ambiguity decorrelation adjustment: a method for fast GPS integer ambiguity estimation[J]. Journal of Geodesy (1995)70:65-82.

[2] Deb K, Pratap A, Agarwal S, et al. A fast and elitist multiobjective genetic algorithm: NSGA-II[J]. Evolutionary Computation, IEEE Transactions on, 2002, 6(2): 182-197.

[3] Hwang C R. Simulated annealing: theory and applications[J]. Acta Applicandae Mathematicae, 1988, 12(1): 108-111.

[4] Dorigo M, Gambardella L M. Ant colony system: a cooperative learning approach to the traveling salesman problem[J]. Evolutionary Computation, IEEE Transactions on, 1997, 1(1): 53-66.

[5] Li X, Shao Z, Qian J. An optimizing method based on autonomous animats: fish-swarm algorithm[J]. System Engineering Theory and Practice, 2002, 22(11): 32-38.

[6] XU Dingjie, LIU Mingkai, SHEN Feng. Fast DGPS Integer Ambiguity Resolution Using Adaptive Genetic Algorithm[J]. Acta Aeronautica et Astronautica Sinica(2013)34:371-377.

[7] Zheng Q H, Zhang Y L. OTF Ambiguity Resolution Based on Genetic Algorithm[J]. Journal of National University of Defense Technology, 2001,23(3): 12-17. 